\title{
Korean Version of the Stroke Rehabilitation Motivation Scale: Reliability and Validity Evaluation
}

\author{
Mina Park, MD, Ji-Yeong Lee, BS, Yeajin Ham, BS, Sang-Wook Oh, MD, Joon-Ho Shin, MD, MS
}

Department of Rehabilitation Medicine, National Rehabilitation Center, Ministry of Health and Welfare, Seoul, Korea

\begin{abstract}
Objective To translate the Stroke Rehabilitation Motivation Scale (SRMS), developed to evaluate the motivation level of stroke patients during rehabilitation, into the Korean language and to verify the reliability and validity of the Korean version of SRMS (K-SRMS).

Methods The K-SRMS was developed following a structured process that included translation, verification, compromise assessment, reverse translation, feedback, and final correction. K-SRMS reliability was evaluated by performing internal consistency and test-retest analyses. The reliability test was conducted in 50 stroke patients. Its validity was assessed by comparing the K-SRMS with the scale and performing exploratory factor analysis. The validity test was conducted in 102 stroke patients.

Results The test-retest analysis showed good reliability, and the internal consistency of the K-SRMS was similar to that of the original version for all, except 4 , items. Thus, these 4 items were excluded, and then the validity test was conducted. Pearson correlation analysis demonstrated that the K-SRMS score was significantly correlated with the BAS total score (Pearson $\mathrm{r}=0.207, \mathrm{p}<0.05$ ). In the exploratory factor analysis, K-SRMS items were categorized into 7 groups (factors), and factors 1 and 4 showed mutual concordance with K-SRMS subscales, including intrinsic motivation factors and amotivation, respectively.

Conclusion The newly developed K-SRMS showed good reliability and validity. It could also be used as a tool to objectify the degree of motivation for rehabilitation among stroke patients in clinical care and research.
\end{abstract}

Keywords Motivation, Reliability and validity, Stroke rehabilitation, Surveys and questionnaires, Translations

\section{INTRODUCTION}

Motivation is one of the key factors affecting the out- come of rehabilitation. When a patient's motivation for rehabilitation is high, the duration of rehabilitation has been reportedly reduced and improvement rates of ac-

Received July 16, 2019; Accepted August 21, 2019

Corresponding author: Joon-Ho Shin

Department of Rehabilitation Medicine, National Rehabilitation Center, Ministry of Health and Welfare, 58 Samgaksan-ro, Gangbuk-gu, Seoul 01022, Korea. Tel: +82-2-901-1884, Fax: +82-2-901-1590, E-mail: asfreelyas@gmail.com

ORCID: Mina Park (http://orcid.org/0000-0002-0996-4522); Ji-Yeong Lee (http://orcid.org/0000-0002-9741-7384); Yeajin Ham (http://orcid.org/00000001-8745-7318); Sang-Wook Oh (http://orcid.org/0000-0001-9493-5277); Joon-Ho Shin (http://orcid.org/0000-0001-6447-8829).

(ㄷ) This is an open-access article distributed under the terms of the Creative Commons Attribution Non-Commercial License (http://creativecommons.org/ licenses/by-nc/4.0) which permits unrestricted noncommercial use, distribution, and reproduction in any medium, provided the original work is properly cited. Copyright $\odot 2020$ by Korean Academy of Rehabilitation Medicine 
tivities of daily living and motor function increased [1,2]. However, anticipating good rehabilitation outcomes is difficult when a patient does not have the motivation and willingness to undergo rehabilitation, which is recommended for a long-term intervention [2]. Therefore, healthcare providers should pay attention in motivating patients for rehabilitation [3].

Previous studies have measured motivation using simple methods, although motivation is diverse. A previous report used only one 5-point scale item (from very depressed to very cheerful) to measure motivation through observation and communication [4]. Another report conducted the assessment based only on the opinion of healthcare professionals and did not consider patients' viewpoint [5]. Moreover, the motivation assessment has been inconsistent among rehabilitation professionals. Therefore, a motivation assessment tool that can objectify a patient's subjective expression and quantify the degree of motivation should be established.

Currently, a few specifically designed assessment tools for motivation have been available in the area of stroke rehabilitation. The Stroke Rehabilitation Motivation Scale (SRMS) is a validated measurement tool for motivation among post-stroke patients that can quantify motivation with objective measurements in the stroke population during the rehabilitation process [6]. When used in another environment, especially in another language, verified translation with consideration of other language characteristics and cultural differences is needed.

Therefore, this study aimed to translate the original SRMS into Korean language and to verify the test-retest reliability and convergent validity of its Korean version (K-SRMS), which can be used as an objective measurement of patient's motivation level in post-stroke rehabilitation. In addition, the study attempted to compare the K-SRMS with the Behavioral Inhibition System/Behavioral Activation System (BIS/BAS) scale, which has not been used in stroke rehabilitation.

\section{MATERIALS AND METHODS}

\section{The SRMS questionnaire}

The original SRMS includes 28 items applicable to stroke rehabilitation, adapted from the Sports Motivation Scale [6]. The Sports Motivation Scale is a motivation assessment tool used in sports according to a theory involv- ing established motivation (self-determination theory). Thus, it reflects the following two types of motivational factors: (1) internal, such as personal satisfaction, enjoyment, and interest; and (2) external factors related to social expectation $[7,8]$. Based on these two types of factors, motivation is further divided into the following 7 subscales in the SRMS questionnaire: (1) extrinsic motivation (EM)-introjected (motivation from external factors leading to internal pressures, such as guilt); (2) EM-regulation (motivation from external pressures or rewards, such as praise from others); (3) EM-identification (motivation from activity participation for personal growth); (4) amotivation (AM; absence of motivation); (5) intrinsic motivation (IM)-knowledge (motivation from knowledge gained with the activity); (6) IM-stimulation (motivation from enjoyment or pleasure of an experience); and (7) IM-accomplishment (motivation from personal satisfaction with activity accomplishment) [6]. Each subscale has corresponding questions. The response to each item is measured on a scale of 1 to 5 . Higher score indicates higher rehabilitation motivation level for all items, except three reverse evaluation items (items \#5, \#12, and \#23), for which a higher score indicates lower motivation level.

\section{The BIS/BAS scale}

The BIS/BAS scale includes 24 items and uses a 4-point Likert score to measure two motivational systems that regulate behavior depending on the responsiveness to punishment (BIS) and reward (BAS) [9]. The BIS is activated by conditional stimuli to avoid aversive outcomes, whereas the BAS involves positive feedback mechanisms to approach stimuli. The BAS scale is divided into the following three subscales: BAS-reward (motivation to achieve pleasant reinforcement in the environment), BAS-drive (motivation to follow a goal), and BAS-funseeking (spontaneous tendency to find novel rewards). The Korean version of the BIS/BAS scale was used in this study [10].

\section{Translation procedure}

The translation process involved linguistic and idiomatic refinement of the original version for Korean translation. Then, a structured questionnaire translation process was performed according to the steps for linguistic validation. Before initiating translation, approval for SRMS translation to the Korean language was obtained from the 
original author Daniel Chan. The first step involved forward translation, performed by two physiatrists, who were professionally fluent in both English and Korean (versions $1.0 \mathrm{a}$ and $1.0 \mathrm{~b}$ ). The second step involved reconciliation of the Korean translation by a translation committee consisting of four members (two physiatrists and two rehabilitation psychologists) who could speak both English and Korean. In this step, the suitability of the Korean translation was verified, and discussions were held for versions 1.0a and $1.0 \mathrm{~b}$ to produce version 1.1. An equivalence between the original version and the Korean version of the SRMS was compromised in this step. The third step involved back translation (reverse translation). A bilingual translator (English and Korean) blinded to the original version translated version 1.1 back to English. Then, the original and reverse translated versions were compared and reviewed to produce version 1.2. The fourth step involved cognitive debriefing and feedback. Version 1.2 questionnaire was applied to 10 people, and the collected feedback indicated that the questionnaire was ambiguous or difficult to understand. After linguistic and idiomatic refinement, version 1.3 was drafted. The final version 1.4 was developed after rectifying the stylistic and spelling-related issues in the preceding versions (Fig. 1).

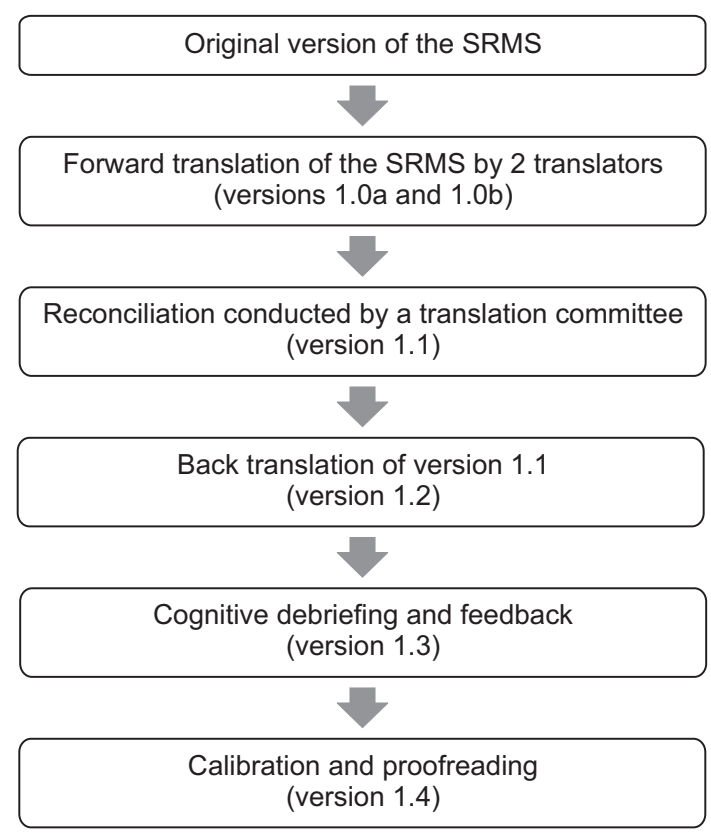

Fig. 1. The translation procedure flow chart of the Korean version of the Stroke Rehabilitation Motivation Scale (SRMS).

\section{Participants}

A total of 102 stroke survivors admitted to the stroke unit of a rehabilitation hospital were enrolled between May 2014 and May 2018. The inclusion criteria were as follows: (1) diagnosis of ischemic or hemorrhagic stroke; (2) cognitive and verbal abilities sufficient to understand and follow researcher's instructions; and (3) agreement to participate after understanding the research contents. The exclusion criteria were as follows: (1) severe comorbidity or disability before the stroke and (2) no consent to participate in this study. Participants' baseline characteristics, including sex, age, stroke type, lesion site, and stroke duration, were assessed. This study was approved by the appropriate Institutional Review Board of National Rehabilitation Center (No. NRC-2014-04-024) and was conducted with written consent from all participants.

\section{Reliability and validity assessment}

To verify the translated K-SRMS, the test-retest reliability was assessed as the first step. The test-retest reliability and internal consistency were measured to establish its reliability. The K-SRMS was assessed twice at 3-day interval among 50 stroke patients. The internal consistency was evaluated to assess the concordance between items within each subscale [11]. Then, its validity was examined by comparing it with the Korean version of the BAS/ BIS scale, and exploratory factor analysis was performed in 102 stroke patients.

\section{Statistical analysis}

For baseline characteristics, absolute values are used to present data for categorical variables, and means with standard deviations are used to present data for numerical variables. Data were analyzed with different approaches. First, Cronbach's alpha was used to evaluate internal consistency and test-retest reliability. Second, Pearson correlation test involving the BIS/BAS scale was used to assess validity. Third, exploratory factor analysis was performed to identify underlying relationships among KSRMS items. All statistical analyses were performed using SPSS version 20.0 (IBM Corp., Armonk, NY, USA). A p-value less than 0.05 was considered statistically significant.

\section{RESULTS}

The reliability test was performed in 50 patients (36 
Table 1. Internal consistency and test-retest reliability of the Korean version of the Stroke Rehabilitation Motivation Scale (SRMS) using Cronbach's alpha

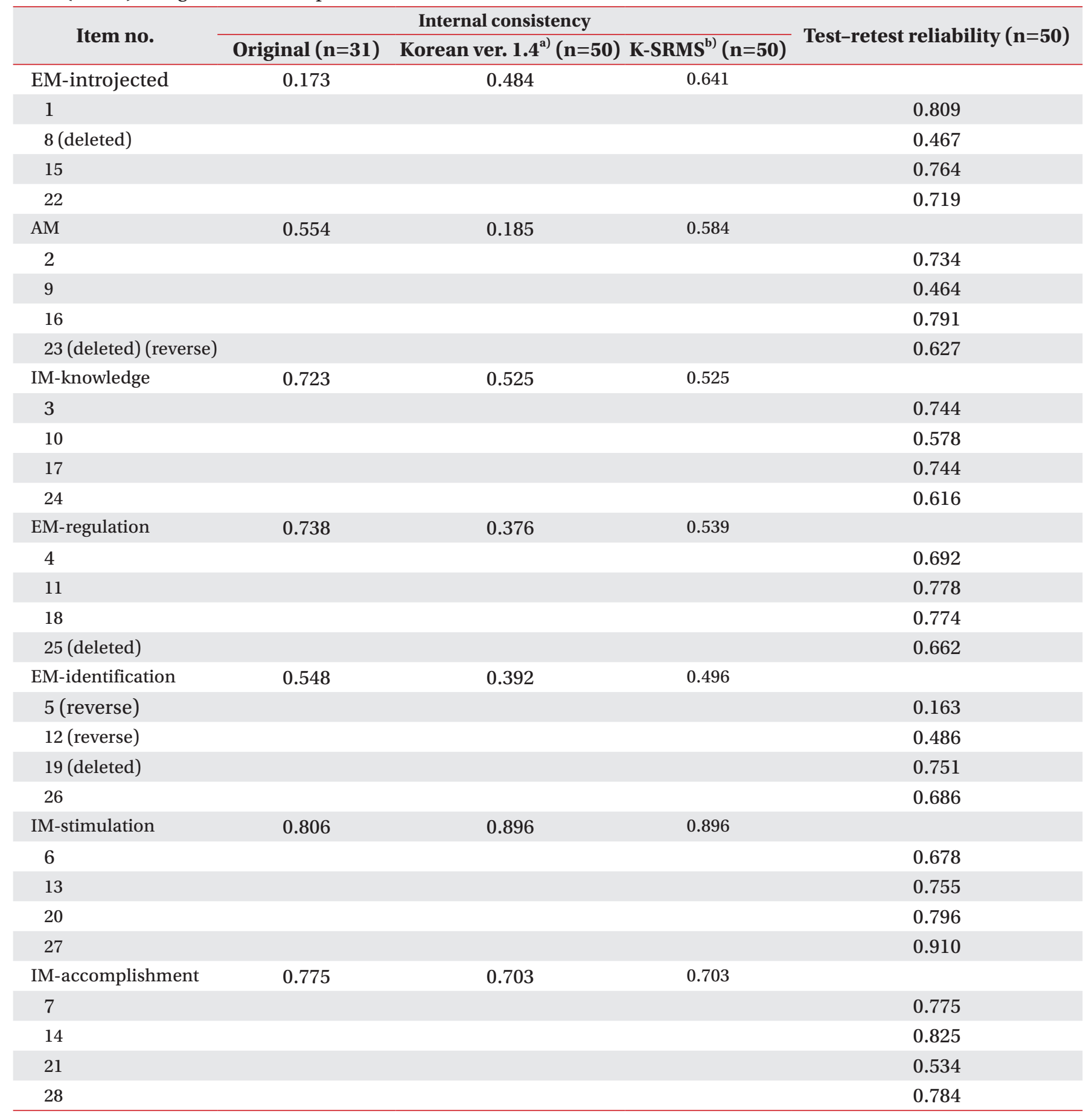

The numbers are the question numbers of the original version. Items \#8, \#19, \#23, and \#25 were excluded after the reliability test. Items \#5, \#12, and \#23 were reverse.

EM, extrinsic motivation; AM, amotivation; IM, intrinsic motivation.

a) 28 items, ${ }^{\text {b) }} 24$ items. 
males; mean age, $52.4 \pm 10.3$ years), and internal consistency and test-retest reliability were assessed. Among the 50 patients, 32 had ischemic stroke and 27 had a right brain lesion. The mean time after stroke was $8.3 \pm 6.2$ months. Subsequently, the validity test was performed in 102 patients (74 males; mean age, $52.6 \pm 11.1$ years). Among the 102 patients, 61 had ischemic stroke and 56 had a right brain lesion. The mean time after stroke was $8.5 \pm 6.9$ months. No patients dropped out or were lost during tests.

\section{Reliability of the SRMS}

The initial internal consistency of the K-SRMS (version 1.4) with 28 items was lower than that of the original SRMS in a few subscales, because 4 items made the internal consistency of each subscale insufficient. Therefore, these 4 items (items \#8, \#19, \#23, and \#25) were excluded, and the remaining 24 items were extracted from version 1.4. These extracted 24 items were grouped as the KSRMS (Appendix 1). Then, an acceptable internal consistency was achieved when compared with the original SRMS (Table 1). With regard to the test-retest reliability of

Table 2. Internal consistency of the BIS/BAS scale using Cronbach's alpha in 50 stroke patients

\begin{tabular}{lcl}
\hline BIS/BAS subscale & Cronbach's alpha & \multicolumn{1}{c}{ Item no. } \\
\hline BAS-reward & 0.788 & $4,7,14,18,23$ \\
\hline BAS-drive & 0.837 & $3,9,12,21$ \\
BAS-fun seeking & 0.812 & $5,10,15,20$ \\
\hline BIS & 0.722 & $2,8,13,16,19,22,24$ \\
\hline
\end{tabular}

BAS, Behavior Activation System; BIS, Behavior Inhibition System. the K-SRMS, Cronbach's alpha was above 0.5 for all items, except 3 items (items \#5, \#9, and \#12). In contrast, with regard to the internal consistency of the Korean BIS/BAS scale, Cronbach's alpha was $>0.7$ for all four subscales (Table 2).

\section{Validity of the SRMS}

Table 3 shows results of the validity analysis that included 102 patients. Pearson correlation test showed that the K-SRMS total score was significantly correlated with the BAS total score (Pearson $\mathrm{r}=0.207, \mathrm{p}=0.037$ ) but not with the BIS total score (Pearson $\mathrm{r}=0.099, \mathrm{p}=0.323$ ). Majority of K-SRMS subscale scores were significantly correlated with BAS subscale scores and BAS total score but not with the BIS total score. Only EM-regulation and EM-introjected scores were significantly correlated with the BIS total score. EM-regulation, which is related to external reward, was significantly correlated with all BAS subscales and the BIS scale. IM-stimulation was significantly correlated with all BAS subscales, but not with the BIS scale. AM was negatively correlated with all BAS subscales and the BIS scale.

In the exploratory factor analysis, variables were categorized into 7 groups (factors), and this number was equal to the number of SRMS subscales; however, the groups did not perfectly match the subscales. The explained total variance was $65.93 \%$, Kaiser-Meyer-Olkin (KMO) score was 0.811 , and Bartlett's sphericity was 0.000 ; therefore, the findings were considered appropriate (Table 4). Results of the commonality test demonstrated that no item needed to be excluded (all 24 items $>0.04$ ).

Table 3. Pearson correlation test of the K-SRMS and Korean BIS/BAS scale ( $\mathrm{n}=102)$

\begin{tabular}{lcccccccc}
\hline & $\begin{array}{c}\text { EM- } \\
\text { introjected }\end{array}$ & $\begin{array}{c}\text { EM- } \\
\text { regulation }\end{array}$ & $\begin{array}{c}\text { EM- } \\
\text { identification }\end{array}$ & AM & $\begin{array}{c}\text { IM- } \\
\text { knowledge }\end{array}$ & $\begin{array}{c}\text { IM- } \\
\text { stimulation }\end{array}$ & $\begin{array}{c}\text { IM- } \\
\text { accomplishment }\end{array}$ & $\begin{array}{c}\text { K-SRMS } \\
\text { total }\end{array}$ \\
\hline BAS-reward & $0.216^{*}$ & $0.359^{* *}$ & $0.210^{*}$ & $-0.220^{*}$ & $0.251^{*}$ & $0.314^{* *}$ & $0.217^{*}$ & $0.221^{*}$ \\
BAS-drive & 0.023 & $0.282^{* *}$ & $0.209^{*}$ & $-0.224^{*}$ & $0.212^{*}$ & $0.341^{* *}$ & $0.235^{*}$ & 0.194 \\
BAS-fun & 0.030 & $0.311^{* *}$ & $0.198^{*}$ & $-0.208^{*}$ & 0.144 & $0.304^{* *}$ & 0.195 & 0.103 \\
BAS total & 0.110 & $0.383^{* *}$ & $0.247^{*}$ & $-0.261^{* *}$ & $0.243^{*}$ & $0.383^{* *}$ & $0.259^{* *}$ & $0.207^{*}$ \\
BIS total & $0.268^{* *}$ & $0.242^{*}$ & 0.085 & -0.151 & 0.184 & 0.170 & 0.100 & 0.099
\end{tabular}

The data are Pearson correlation coefficients.

K-SRMS, Korean version of Stroke Rehabilitation Motivation Scale; BAS, Behavior Activation System; BIS, Behavior Inhibition System; EM, extrinsic motivation; AM, amotivation; IM, intrinsic motivation.

${ }^{*} \mathrm{p}<0.05,{ }^{* *} \mathrm{p}<0.01$, calculated by Pearson correlation test. 
Mina Park, et al.

Table 4. Exploratory factor analysis of the K-SRMS

\begin{tabular}{|c|c|c|c|c|c|c|c|}
\hline \multirow{2}{*}{ Original SRMS item no. } & \multicolumn{7}{|c|}{ Factor (factor loading) } \\
\hline & 1 & 2 & 3 & 4 & 5 & 6 & 7 \\
\hline 13 & 0.830 & - & - & - & - & - & - \\
\hline 27 & 0.783 & - & - & - & - & - & - \\
\hline 20 & 0.752 & - & - & - & - & - & - \\
\hline 14 & 0.749 & - & - & - & - & - & - \\
\hline 6 & 0.661 & - & - & - & - & - & - \\
\hline 15 & 0.631 & - & - & - & - & - & - \\
\hline 7 & 0.538 & - & - & - & - & - & - \\
\hline 1 & - & 0.796 & - & - & - & - & - \\
\hline 3 & - & 0.732 & - & - & - & - & - \\
\hline 21 & - & 0.527 & - & - & - & - & - \\
\hline 11 & - & - & 0.784 & - & - & - & - \\
\hline 10 & - & - & 0.719 & - & - & - & - \\
\hline 4 & - & - & 0.525 & - & - & - & - \\
\hline 28 & - & - & 0.484 & - & - & - & - \\
\hline 16 & - & - & - & -0.697 & - & - & - \\
\hline 2 & - & - & - & -0.620 & - & - & - \\
\hline 17 & - & - & - & 0.538 & - & - & - \\
\hline 9 & - & - & - & -0.445 & - & - & - \\
\hline 12 & - & - & - & - & 0.842 & - & - \\
\hline 5 & - & - & - & - & 0.609 & - & - \\
\hline 18 & - & - & - & - & - & 0.684 & - \\
\hline 24 & - & - & - & - & - & 0.607 & - \\
\hline 22 & - & - & - & - & - & - & 0.841 \\
\hline 26 & - & - & - & - & - & - & 0.659 \\
\hline$\%$ of variance & 30.09 & 38.70 & 45.30 & 51.44 & 56.76 & 61.47 & 65.93 \\
\hline & $\mathrm{MO}=0.81$ & rtlett's t & ii-squar & $9.678, \mathrm{~d}$ & Signific & $=0.000)$ & \\
\hline
\end{tabular}

Items \#8, \#19, \#23, and \#25 were excluded after the reliability test.

K-SRMS, Korea version of the Stroke Rehabilitation Motivation Scale; KMO, Kaiser-Meyer-Olkin scale; df, degree of freedom.

\section{DISCUSSION}

We established the K-SRMS with 24 items after removing 4 items that exhibited insufficient internal consistency. Thus, its reliability was acceptable. However, assessing the inter-relationships among the 4 items was difficult because each item was in a different subscale. Cronbach's alpha values of the 7 K-SRMS subscales were comparable to those of the original SRMS, indicating that the translation process successfully maintained the intention and characteristics of the original SRMS [6]. Moreover, Cronbach's alpha of EM-introjected increased from 0.173 in the original SRMS to 0.641 in the K-SRMS, and therefore, EM-introjected was better in the K-SRMS. The K-SRMS demonstrated a good test-retest reliability for all, except 3, items. The lowest test-retest reliability was noted for item $\# 5$, which might be related to the fact that the question for this item was easily affected by the caregiver or healthcare professional who wants patients to be treated, as this study was performed among stroke patients admitted to a rehabilitation hospital.

A concurrent validity analysis for the K-SRMS and the BIS/BAS scale was performed. The original SRMS did not undergo concurrent validity assessment due to the absence of an established measurement tool for motivation. In addition, it did not show predictive validity with 
mood-relevant measurements (anxiety, depression, and stress) and physical performance measurements (sitto-stand rate and walking distance) [6]. To assess the concurrent validity, the BIS/BAS scale was selected as a comparative motivation measurement tool because it has representative psychometric properties under various conditions [12,13]. Initially, the reliability of the BIS/BAS scale with regard to internal consistency was assessed because no studies have used the BIS/BAS scale in the area of stroke rehabilitation. Then, the relationship between the K-SRMS and BIS/BAS scale was examined. The K-SRMS total score was significantly correlated with the BAS total score, and most BAS subscales were positively correlated with K-SRMS subscales. Only the AM subscale of the K-SRMS was negatively correlated with all BIS/BAS scales. These findings confirmed that the K-SRMS appropriately reflects motivation with regard to behavior-activating factors. Especially, BAS-reward and EM-regulation (motivation from external pressure or rewards), which deal with the same concept (reward), showed the highest correlation. On the contrary, EM-introjected (external motivation activator to lessen embarrassment or guilt) was significantly associated with BIS but not with BAS, indicating that the introjected feeling might lead to motivation by inhibiting one's behavior. Thus, the K-SRMS could be considered to involve mainly BAS-relevant and BIS-relevant factors, and this mutual concordance between the K-SRMS and BIS/BAS scale could solidify the validity of both scales.

An exploratory factor analysis was performed to explore the K-SRMS characteristics among stroke patients. Commonality and a similar tendency with regard to the original SRMS were observed, and 7 groups (factors), which were similar to the 7 subscales of the original SRMS, were identified. However, the groups did not perfectly match the subscales. All 4 IM-stimulation items (items \#6, \#13, $\# 20$, and \#27) were grouped together as factor 1 , and all 3 amotivation items (items \#2, \#9, and \#16) were grouped as factor 4. Factor 1, including the most important set of questions in the K-SRMS, accounted for $30.09 \%$ of the total variance and included all IM-stimulation items, 2 IM-accomplishment items (items \#7 and \#14), and 1 EMintrojected item (item \#15). These findings indicate that intrinsic rather than extrinsic factors have major roles, especially with regard to enjoyment or satisfaction, in stroke rehabilitation motivation.
Recently, motivation has been emphasized as an essential part of stroke rehabilitation, thus supporting the importance of virtual reality or game-based rehabilitation $[14,15]$. However, motivation has not been properly evaluated, and instead, other areas, such as emotion and fun, have been assessed using stroke impact scales or usability tests due to the absence of a suitable measurement tool for motivation $[15,16]$. The K-SRMS enables direct assessment of motivation per se, allowing evaluation of the effects of a specific intervention on motivation. Additionally, the K-SRMS could be used to determine whether motivation, as a trait of each participant, predicts the outcomes of stroke rehabilitation. Furthermore, the KSRMS could be used in not only a clinical setting but also a research setting because it includes detailed subscales representing diverse features.

This study has some limitations. First, the survey was conducted at a single rehabilitation hospital with, mainly, subacute or chronic patients, and characteristics of the acute phase of stroke rehabilitation were not identified. Second, the study did not assess other outcome measures, including physical and neuropsychological measures such as Fugl-Meyer Assessment or Beck Depression Inventory; therefore, its relationships with other outcome measures were not assessed, and the predictive validity not evaluated. Further studies evaluating both predictive and concurrent validity in a larger population might be needed.

In conclusion, this study demonstrated the reliability and validity of the K-SRMS, which was translated with a verified process, in order to evaluate the motivation for stroke rehabilitation. The K-SRMS with 7 subscales allows objective and comprehensive evaluation of motivation for stroke rehabilitation in Korean patients. This scale could be applied in stroke rehabilitation to help optimal treatments.

\section{CONFLICT OF INTEREST}

No potential conflict of interest relevant to this article was reported.

\section{ACKNOWLEDGMENTS}

This research was funded by the Translational Research Center for Rehabilitation Robots, National Rehabilitation 
Center, Ministry of Health and Welfare, Republic of Korea (Grant number: NRCTR-IN16002, NRCTR-IN17002).

\section{AUTHOR CONTRIBUTION}

Conceptualization: Shin JH. Methodology: Shin JH. Formal analysis: Lee JY. Project administration: Lee JY, Ham YJ. Visualization: Park MA, Oh SW. Writing - original draft: Park MA, Shin JH. Writing - review and editing: Park MA, Shin JH. Approval of final manuscript: all authors.

\section{REFERENCES}

1. Langhorne P, Holmqvist LW; Early Supported Discharge Trialists. Early supported discharge after stroke. J Rehabil Med 2007;39:103-8.

2. Maclean N, Pound P, Wolfe C, Rudd A. Qualitative analysis of stroke patients' motivation for rehabilitation. BMJ 2000;321:1051-4.

3. Maclean N, Pound P, Wolfe C, Rudd A. The concept of patient motivation: a qualitative analysis of stroke professionals' attitudes. Stroke 2002;33:444-8.

4. Tupper A, Henley S. Predictive factors in stroke outcome and implications for intervention. Int J Rehabil Res 1987;10:119-22.

5. Resnick B. Motivation in geriatric rehabilitation. Image J Nurs Sch 1996;28:41-5.

6. White GN, Cordato DJ, O'Rourke F, Mendis RL, Ghia D, Chan DK. Validation of the Stroke Rehabilitation Motivation Scale: a pilot study. Asian J Gerontol Geriatr 2012;7:80-7.

7. Pelletier LG, Tuson KM, Fortier MS, Vallerand RJ, Briere NM, Blais MR. Toward a new measure of intrinsic motivation, extrinsic motivation, and amotivation in sports: the Sport Motivation Scale (SMS). J Sport Exerc
Psychol 1995;17:35-53.

8. Mallett C, Kawabata M, Newcombe P, Otero-Forero A, Jackson S. Sport Motivation Scale-6 (SMS-6): a revised six-factor sport motivation scale. Psychol Sport Exerc 2007;8:600-14.

9. Scholten MR, van Honk J, Aleman A, Kahn RS. Behavioral inhibition system (BIS), behavioral activation system (BAS) and schizophrenia: relationship with psychopathology and physiology. J Psychiatr Res 2006;40:638-45.

10. Kim KH, Kim WS. Korean-BAS/BIS scale. Korean J Health Psychol 2001;6:19-37.

11. Streiner DL. Starting at the beginning: an introduction to coefficient alpha and internal consistency. J Pers Assess 2003;80:99-103.

12. Jorm AF, Christensen H, Henderson AS, Jacomb PA, Korten AE, Rodgers B. Using the BIS/BAS scales to measure behavioural inhibition and behavioural activation: factor structure, validity and norms in a large community sample. Pers Individ Dif 1998;26:49-58.

13. Johnson SL, Turner RJ, Iwata N. BIS/BAS levels and psychiatric disorder: an epidemiological study. J Psychopathol Behav Assess 2003;25:25-36.

14. Laver KE, Lange B, George S, Deutsch JE, Saposnik G, Crotty M. Virtual reality for stroke rehabilitation. Cochrane Database Syst Rev 2017;11:CD008349.

15. Shin JH, Ryu H, Jang SH. A task-specific interactive game-based virtual reality rehabilitation system for patients with stroke: a usability test and two clinical experiments. J Neuroeng Rehabil 2014;11:32.

16. Shin JH, Kim MY, Lee JY, Jeon YJ, Kim S, Lee S, et al. Effects of virtual reality-based rehabilitation on distal upper extremity function and health-related quality of life: a single-blinded, randomized controlled trial. J Neuroeng Rehabil 2016;13:17. 
Appendix 1. Korean version of the Stroke Rehabilitation Motivation Scale (K-SRMS)

뇌졸중 재활 동기 척도 24 항목

이름

등록 번호:

평가일:

년 월 일

아래에 제시된 설문이 당신이 재활에 참여하는 이유를 얼마나 반영하는지 알고자 합니다. 예, 아니오가 아닌 아래의 척도로 대답해주세요.

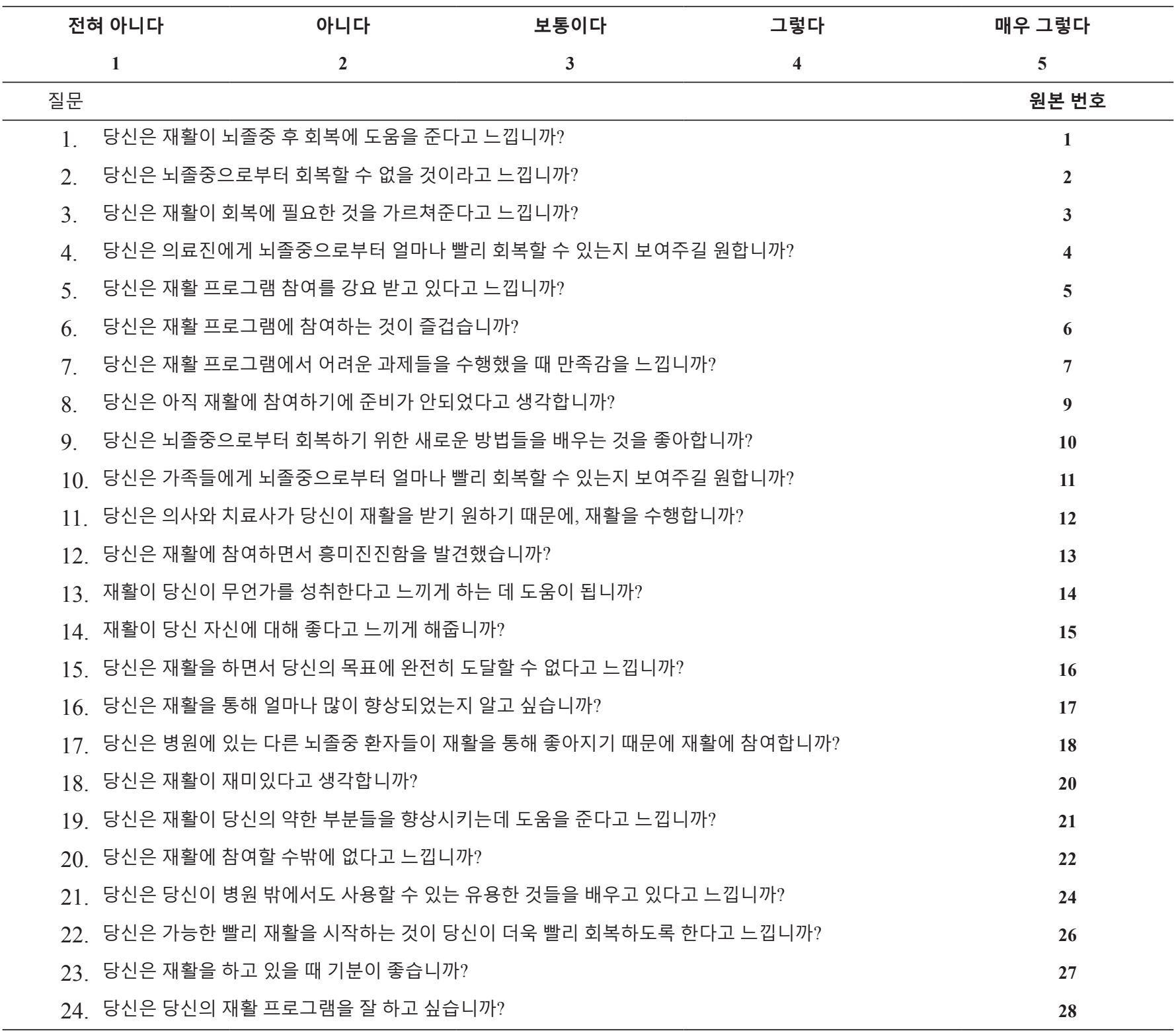

